\title{
Design of Senior Family Bathroom System Based on Demand Theory
}

\author{
Rong $\operatorname{Han}^{1}$, Dandan Shao ${ }^{2 *}$ and YuXin Wang ${ }^{3}$ \\ ${ }^{1}$ College of Art, Jiangsu University, Zhenjiang City, Jiangsu Province,212000, China \\ ${ }^{2}$ College of Art, Jiangsu University, Zhenjiang City, Jiangsu Province,212000, China \\ ${ }^{3}$ College of Art, Jiangsu University, Zhenjiang City, Jiangsu Province,212000, China
}

\begin{abstract}
Aiming at the characteristics of the elderly population, based on the demand theory, this paper proposes a design scheme of a suitable family bathroom system. Based on the theory of Maslow's demand theory, understand the real psychological status of the elderly through theoretical transformation, analyze the physical and psychological characteristics of the elderly, and simulate the specific behavior of the elderly in the bathroom space system through user interviews. In response to the elderly's needs for the space, facilities and supporting facilities of the bathroom system, a type of bathroom space was finally selected to carry out the design practice, which provided certain theoretical support and practical reference value for the subsequent research of bathroom products for the elderly.
\end{abstract}

\section{Introduction}

Since 2000, China has begun to enter an aging society. Population aging is an inevitable trend in social development and a hot topic in the world today (as shown in Figure 1). After entering old age, the physiological functions of the human body will also degrade accordingly. Athletic young people, seniors have different demands for bathroom systems. At present, developed countries 'research on bathroom systems for the elderly is very mature. For example, Japan and Germany, which have more developed industrial designs, attach more importance to the design of barrier-free facilities for the elderly. Many elderly and disabled works have been born. especially in Japan, where the degree of aging is more serious, the research results are extremely advanced. In contrast, the research on the bathroom system for the elderly in China is still in its infancy. Although many universities and institutions have researched the products for the elderly, most of
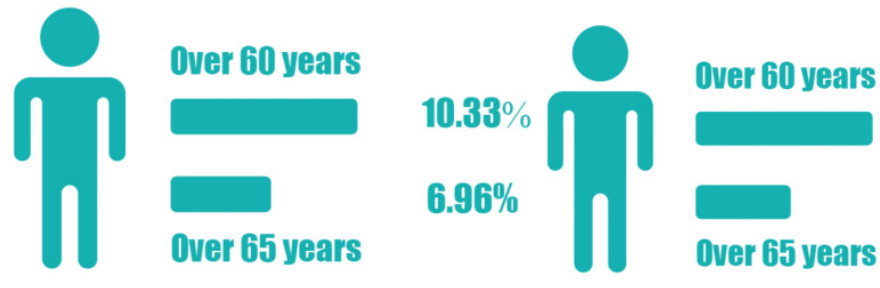

$13.26 \%$

8.87\%

them focus on the general analysis of the product size and lack the corresponding in-depth exploration. At present, there are many plagiarism products in the bathroom market and lack of innovative design. The design of home bathroom systems based on the theory of needs is a targeted, humanized adjustment and change based on comprehensive consideration of the physical and psychological needs of the elderly. It can not only provide the basic bathroom needs of the elderly, but also give the elderly perfect and comfortable bathroom experience.

The physical condition of the elderly is very different in various aspects. According to the survey report of the United Nations World Health Organization, it can be divided into the following three levels (as shown in Figure 2). This article restricts the main research object to the first-class aging healthy elderly For other types of elderly people, only a few mentions were made in the analysis without focusing on the research. The final practice is also aimed at healthy elderly people. Wheelchair users are within the scope of this study.

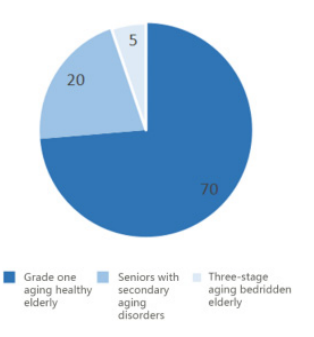

Figure 1. Proportion of population in the fifth and sixth national census

Figure 2. Senior group classification 


\section{Demand Theory}

"Need" refers to the quantity of goods that consumers themselves are willing and able to purchase over a given price within a certain period of time. Most people make driven by desire. In 1943, Abraham Maslow put forward the theory of Maslow's hierarchy of needs in The Theory of Human Motivation. He believes that people have needs and can be divided according to levels, from low to high and level by level. The easiest to meet is physiological needs, and the hardest to achieve is selfrealization.

This article is mainly based on the research of Maslow's hierarchy of needs theory, which divides the elderly's psychological and physical need of bathrooms into levels. This theoretical transformation is helpful to understand the true mental state of the elderly. The following is a detailed description of the needs of seniors at all levels into the need for sanitary systems.

(1) Physiological needs — - is the most basic needs, which means that the bathroom system needs to meet a series of basic physiological needs such as the metabolism, hygiene, and cleanliness of the elderly [1], and is also the basis for achieving the needs.

(2) Safety needs — the same is a low-level need. The elderly gradually slow down due to the decline of their own body functions, so the need for safety is more serious [2]. Therefore, the importance of safety needs for the bathroom system is self-evident. Is also a basic attribute that all products should meet.

(3) Social needs — Needs at a higher level are a manifestation of the elderly's pursuit of quality of life. The elderly in life pay more attention to the sense of belonging of fallen leaves and roots. Attach great importance to the product design of the elderly and the improvement of their quality of life are also expressions of caring for the elderly, so that the elderly have a feeling of trust and warmth in the bathroom space [3].

(4) Be respected - a higher level of need. This includes both personal feelings of achievement or selfworth, as well as recognition and respect from others. The connection between the elderly group and the bathroom system is reflected in the designer's deep understanding of the elderly to make the bathroom system more intimate and humane, so that the elderly feel respected, recognized and trusted.

(5) Self-actualization needs__ are the highest level of needs. A very prominent point in the psychological problems of the elderly is inferiority. As the opportunity to prove themselves gradually disappears, a sense of desolation will emerge. In the bathroom system, it can be understood that the independently completed bathing experience can stimulate the potential of the elderly, make them more independent, and stimulate their passion for life.

\section{Research on the Needs of Sanitary Bathroom for the Elderly}

In China, people over 60 years can be divided into four stages. The age of 60-64 is a healthy and active period, the age of 65-75 is a period of self-care, the age of 75-84 is a period of self-care decline, and the age of 85 or more is a period of cared [4]. The main research object of this study was 60-75 years old.

\subsection{Analysis of the physical characteristics of the elderly}

With the increase of age, the decline of the physical fitness of the elderly is reflected in three aspects: the degradation of the sensory system, the degradation of the motor system, and the degradation of the nervous system. The sensory system mainly refers to vision and hearing; Most of the time the elderly vision will loss. In places with poor light, vision is blurred and presbyopia, which will cause the elderly to fall to the toilet. Hearing degradation is mainly manifested in slow response to noisy low-frequency sounds. Speakers need to increase volume, and close to their ears to hear. The motor system mainly refers to height, bones, and kidneys (urinary system); research shows that when you enter old age, your height will slowly shrink, usually dropping about $3 \mathrm{~cm}$. Second, the elderly are prone to calcium deficiency, their bones are fragile, and minor impacts can cause significant damage to the body. And with the decline of the kidney and urinary system, the frequency of urinary frequency in the elderly is more serious. Finally, with the aging of the elderly's nervous system, the elderly's ability to recognize and adapt to new things is weak, and their limbs and body balance are also poor.

\subsection{Analysis of the psychological characteristics of the elderly}

The psychological needs of the elderly can be divided into five categories (as shown in Figure 3): dependence on lover, belonging and stability, interpersonal communication, respect and self-actualization. Among them, the dependence on relatives refers to the traditional Chinese seniors' pursuit of children and grandchildren. Belonging and stability refer to the old people's nostalgia. The old familiar things make the old people stable and have a sense of belonging. Interaction means that the elderly hope to have more communication and communication with the outside world and enjoy the same opportunities as young people to share their inner world emotions with others. To be respected means that they are afraid that their aging will bring a series of troubles and burdens to their families, and they are eager to have the ability to be independent and thus be respected by others. Self-realization refers to the fact that the elderly are actually helpless about getting older. They are afraid of being disconnected from society and hope to realize their own value as young, and be recognized and favored by others. 
In summary, we can summarize the negative attitudes of the elderly are fear of loneliness and easy inferiority. These bad emotions will have a bad impact on the lives of the elderly, and sanitary products with caring meaning can greatly ease these bad emotions. Therefore, comprehensive consideration should be taken when designing the bathroom system, while meeting the physiological and psychological needs.

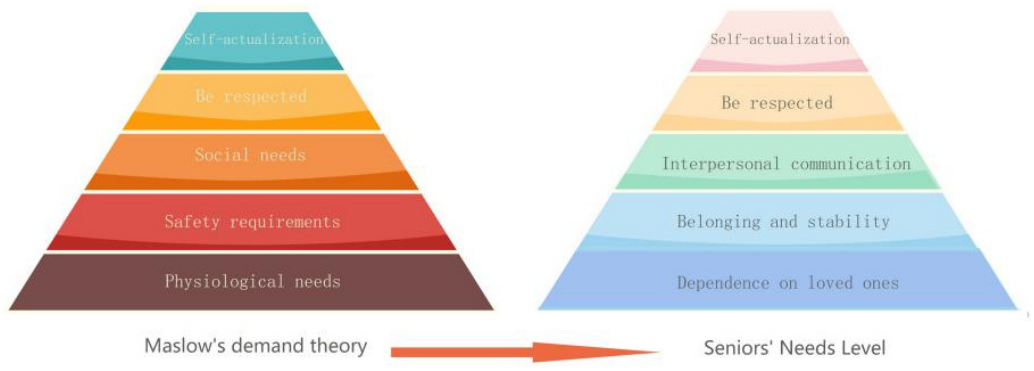

Figure3. psychological characteristics of the elderly

\subsection{Behavioral analysis of elderly people in bathroom space}

Through interviews with multiple healthy old people and the designer's simulation of the bathroom behavior of the elderly, the bathroom behavior of the elderly can be divided into three types: shower behavior, toilet behavior, and washing behavior. When using the toilet, squatting is used as the main method. Supporting armrests must be provided on both sides of the toilet. The elderly need fixed-point support when squatting, and they need to support the fulcrum when they get up. Washing ibehavior mainly include brushing teeth and washing face, the actions cover standing, bending over, turning, etc. The elderly have a poor sense of balance and are prone to unstable instability. The side of the washing table needs to provide assistance for standing for a long time. Bathing behaviors include shampooing, bathing, changing clothes, etc. There are many behaviors in this space, including standing, squatting, getting up, bending over, raising hands, etc. The following figure is a specific analysis of the bathing behavior of the elderly. Both the balance and physical strength are weak, the elderly should not stand for a long time. Safety shower chair should be added in the shower room. Secondly, the elderly still need to rely on external forces to slowly move when turning around, so handrails should be installed in the shower room at appropriate positions. In addition, it is necessary to pay attention to the behavioral connectivity between the various spaces, which must meet the needs of the elderly's single behavior and the elderly's movement law.

\subsection{Questionnaire survey of bathroom system for the elderly Conclusion}

In this project, a questionnaire survey was conducted for a healthy self-care elderly aged 55-70 in a community in Wuxi. A total of 100 questionnaires were distributed and 73 samples were recovered, including 38 males and 35 females (as shown in Figure 4). According to statistics: (1) At present, there is only one bathroom in the elderlys' home, with an area of 4-6 square meters accounting for $53 \%$. A small numbers have two bathrooms. $70 \%$ of the elderly expressed dissatisfaction with the bathroom system and facilities at home, Some bathroom products cannot meet the needs of elderly user groups. The results of the questionnaire report show that the prominent problems of the elderly in the bathroom facilities are mainly reflected in: $55 \%$ of the elderly think that the bathroom floor is slippery and there are hidden safety risks, $34 \%$ of the elderly think that the bathroom space is not wet and dry; $17 \%$ of the elderly think The floor is easy to accumulate water. $10 \%$ of the elderly think that the bathroom is too dark. $16 \%$ of the elderly said squatting was inconvenient; $7 \%$ of the elderly responded poorly to ventilation; $20 \%$ of the elderly think it is inconvenient to Take the clothes from the fixed thing frame ; elderly people want to add bathroom facilities: $57 \%$ of elderly people think that safety handrails should be added; $37 \%$ of elderly people think that bathrooms should add distress alarms; $33 \%$ of people think that shower safety Seatss hould be added; $21 \%$ of seniors believe that lower positions shower heads stents should be added ; $12 \%$ of seniors want to add heating and ventilation facilities in bathrooms; $18 \%$ of seniors think that night sensor lights need to be added; $33 \%$ of seniors think Hangers and shoe racks should be added; the elderly think that the important order of the bathroom are safety, privacy, cleanness, convenience, health, etc.

\section{Research on Senior Family Bathroom Systems Based on the Needs of Seniors}

\subsection{Space research of home bathroom system}

Bathroom space can be divided into small $\left(2.5-4 \mathrm{~m}^{2}\right)$ bathroom space, medium-sized bathroom space $\left(4-8 \mathrm{~m}^{2}\right)$ and large bathroom space (greater than $8 \mathrm{~m}^{2}$. Among them, the bathroom space of more than $5 \mathrm{~m}^{2}$ is more satisfactory for most elderly people. In addition to basic sanitary ware, it can also meet the needs of wet and dry division of bathroom space. The shower space is centered on bathtubs and shower facilities, which include shampooing, bathing and changing clothes. Compared with bathtubs, showers are more convenient, watersaving and safe, and are more popular among seniors. The toilet space is centered on the toilet. Due to the 
degradation of the physical functions of the elderly, their physical appearance is different from that of the young people. The size requirements of the toilet space are also different. The minimum functional space of the elderly who is completely self-care is approximately $900 *$ $1400 \mathrm{~m}^{2}$ [5]. At the same time, the distance between the front of the toilet and the toilet must be more than $500 \mathrm{~mm}$. The main facilities of the washroom are washbasins, faucets and other bathroom furniture. The elderly usually require the distance between the washbasin and the wall to be above $600 \mathrm{~mm}$ when doing the face washing. It can prevent the elderly from being injured by bending over. At the same time, there must be enough space under the washbasin to place legs. The width of the washbasin should be greater than $900 \mathrm{~mm}$ [6].

\subsection{Analysis of Home Bathroom System Facilities}

Bathroom facilities include toilets, washstands, bathtubs and shower rooms; the elderly's waist and leg functions are reduced, it is more difficult to get up and squat, and standing or squatting for a long time is prone to lose balance and fall. The toilet is more suitable for sit type seat implement. The height is relatively high to reduce the burden on the legs of the elderly. There are three types of countertops: counter type, stand type and wall type. The counter type is most suitable for the elderly [7]. When the elderly are standing and washing their faces, the height of the washstand should be $750-850 \mathrm{~mm}$. For the elderly using a wheelchair, the height of the washstand is $650-750 \mathrm{~mm}$. In summary, the height of the washstand designed for the elderly should be between $650-850 \mathrm{~mm}$. The shower room includes shower column, faucet and other related equipment. The division of dry and wet space can not only reduce the elderly's slipping events, but also can prevent splashing and heat preservation. The tempered glass material can effectively protect the personal safety of the elderly. In order to make it easier to place the shower head, the head fixing devices of different heights should be designed. The floor is mainly made of non-slip material to reduce slip accidents.

Bathroom auxiliary facilities include bath chairs, armrests, emergency call systems, etc. These devices can effectively ensure the personal safety of the elderly [8]. prolonged standing bathing is prone to breathing difficulties, dizziness, etc. Therefore, a bathing chair must be provided, which can be fixed in a suitable position on the wall and can be folded to save space. The setting of the armrest can be used to maintaining balance, supporting weight, relying on labor-saving, short-term body relying on force to support, pushing hands in hand, and supporting. There are three main types of handrails in the bathroom. L type U type and T type. Stainless steel PE plastic and ABS plastic steel are resistant to corrosion and difficult to deform [9].suitable for long-term use of the elderly, at the same time The surface texture of the roof, spirals and particles can increase the friction and play a non-slip effect. Different types of armrests have different stress points and application points. $\mathrm{T}$ and $\mathrm{L}-$ shaped armrests can provide support and force assistance. They are suitable for use around the toilet. Vertical Ushaped armrests are suitable for installation on the outer edge of the shower room. In addition, the emergency call system is also a necessary device. In the event of an accident, the elderly can be rescued in a timely manner. For the convenience of use, it is usually installed on the side wall within easy reach of the elderly. The height from the ground is $900 \mathrm{~mm}$ and the distance from the rear wall is $750 \mathrm{~mm}$.

\subsection{Research on supporting facilities of home bathroom system}

With the degradation of the elderly's urinary system, the frequency of using toilets has gradually increased. At present, the toilets in most homes are backlit and the light is low. In addition, the elderly have poor eyesight. Accidents such as slipping in the bathroom often occur. To avoid this danger, we should pay attention to the design of the lighting system. The overall lighting should be as soft as possible to avoid strong light to protect the eyesight of the elderly. Incandescent and fluorescent lights are the mainstays. Local lighting can be used in the washbasin area. It is best to add adjustable The secondary mirror with magnifying effect is convenient for the elderly. In addition, the night lighting facilities should be added. The elderly have poor night vision and it is difficult to find the switch position at night. It is necessary to add the induction light in the bathroom space where it is prone to occur bumps. In view of the poor immunity of the elderly, fear of cold and wind, the bathroom needs to be equipped with heating facilities. It is best to use electric heating under the ground or infrared radiation to ensure safety; in addition, mechanical ventilation is required to decrease the probelem of chest tightness and dizziness. The mechanical ventilation device can quickly remove hot air in time to ensure normal breathing.

\section{Design practices of home bathroom systems for the elderly}

\section{1 design positioning}

The research object of this article is the elderly in selfcare and healthy period. At the same time, the size of the shower room selected is 5-6 square meters. Through the above research on the needs of the elderly, the final design goals and design value positioning are: Level 1 requirements; meeting basic safety requirements, that is, to help them improve their bathing experience from the perspective of safety and comfort; Level 2: Improve selfcare ability Experience, which encourages them to bathe alone; Level 3: Self-esteem. improve the dignity of the elderly when bathing (as shown in Figure 5). The higher the level, the more difficult it is to complete. The highlevel bathing experience must be achieved on the basis of the completion of the low-level experience. 


\begin{tabular}{|l|l|l|}
\hline problems in bathroom systems & People & percentage \\
\hline Slippery ground & 40 & 55 \\
\hline No wet and dry separation & 24 & 34 \\
\hline Water easily accumulates on the ground & 12 & 17 \\
\hline Squatting inconvenience & 11 & 16 \\
\hline Dim light & 7 & 10 \\
\hline Poor ventilation & 5 & 7 \\
\hline Inconvenient to hold clothes on the & 14 & 20 \\
\hline fixed rack & & percentage \\
\hline Hope to add bathroom facilities & People & 57 \\
\hline Safety handrail & 41 & 37 \\
\hline Call for siren & 27 & 33 \\
\hline Shower seat & 24 & 21 \\
\hline Low-level shower head & 15 & 12 \\
\hline Heating and ventilation facilities & 8 & 18 \\
\hline Night sensor light & 13 & 9 \\
\hline Hanger for bathing & 6 & \\
\hline
\end{tabular}

Figure 4. Survey results

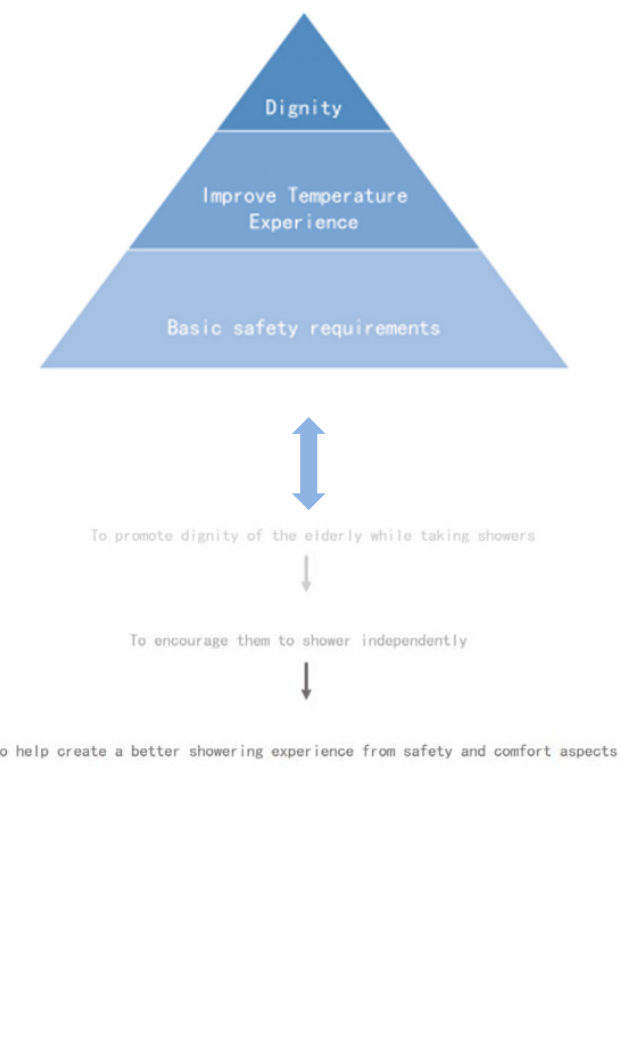

Figure 5. Design positioning complex ecosystem

\section{2design instructions:}

The elderly are slow to move and physically inconvenient. In order to minimize the elderly's movement distance, the most commonly used toilets, shower rooms, and washstands are arranged in an arc. Considering the phenomenon which most elderly people feedback that the bathing space is too small, and prone to bumps. The arc-shaped space setting can maximize the bathing area, and compared to the linear structure, the arc type can give the elderly a safer psychological suggestion, and can significantly reduce bump injuries when falling.

The display instrument panel of the shower uses an intelligent control system. Due to the poor strength of the elderly, the display interface is simplified as much as possible to make the interface information clear. The instrument panel is mainly divided into two functional areas, the space temperature control area and the water temperature control area. The elderly The bath temperature can be adjusted by rotating the two buttons. The glass interface is coated with waterproof and antifog material to keep the interface clear to the maximum. There is a voice dialing device below, the elderly can directly make emergency calls by pressing the corresponding person button in case of emergency. The entire device is retro style. Cater to the retro feelings of the elderly. In addition, a foldable safety seat is added to the shower room. The circular backrest is more ergonomic and can bring the greatest sense of security to the elderly. An anti-fall detection device and an alarm device are arranged at a low place, when an elderly person slips, an emergency rescue can be obtained in time. U-shaped and L-shaped T-shaped handrails are added to both sides of the toilet and the washstand, which is convenient for the elderly to perform lifting, turning, walking and other actions [10]. In order to solve the problem that the elderly take out their clothes when taking a bath, a removable clothes dryer is designed separately. It adopts the shape of a suitcase to increase the mobility. Press the switch button, the machine will automatically perform the heating function, the elderly can directly put on warm clothes after taking a bath in winter. In addition, the device can also be used as a heater alone. The wind direction and gear can be adjusted; the lever can also be pulled out for suspension use. The overall light is mainly warm and bright. Local lighting is added at the washstand, and an adjustable mirror with magnification function is set. 


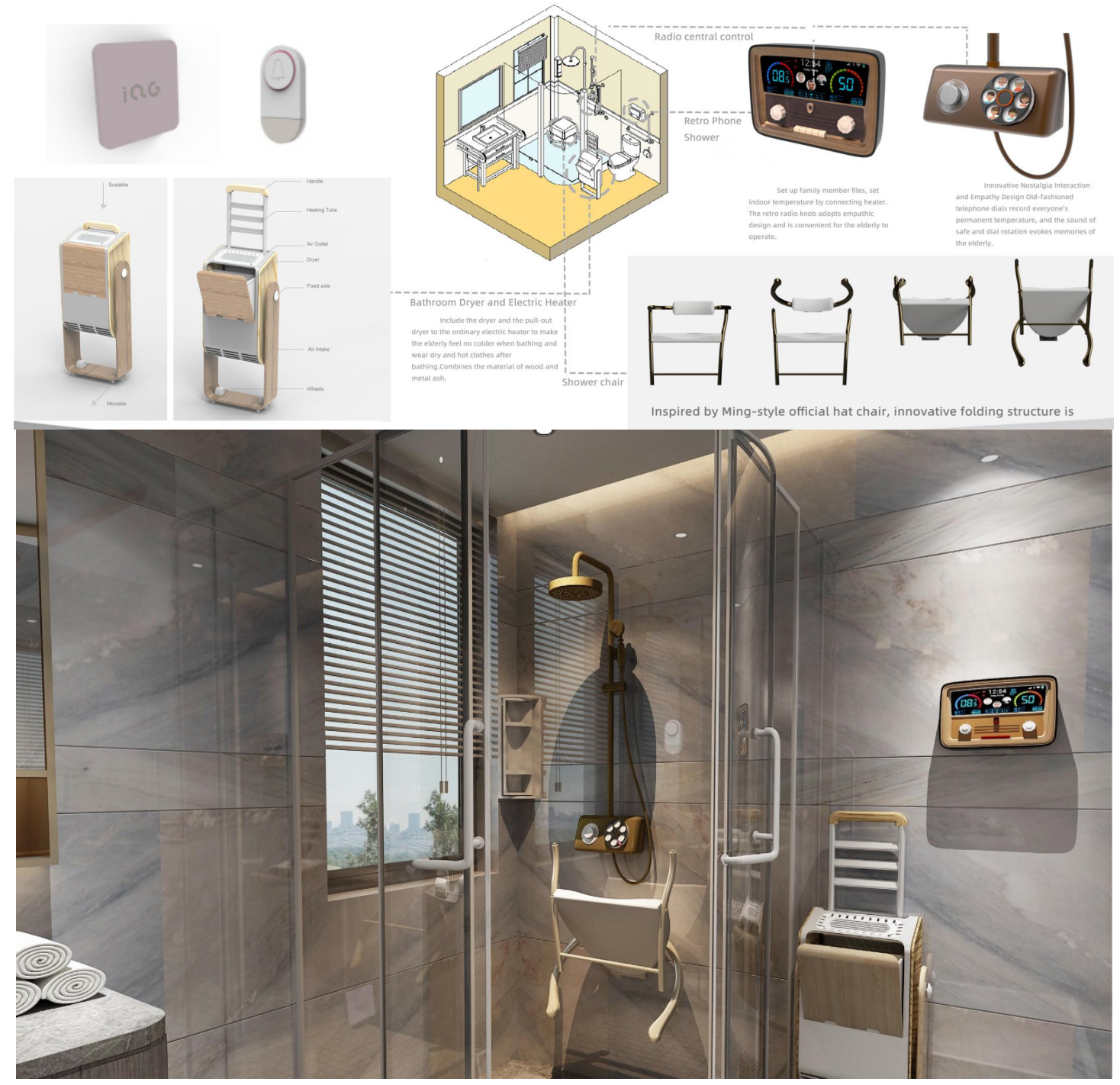

Figure 6. Design case disply

\section{Conclusion}

Bathing activities are common daily activities of the elderly. With the increase of age, the physiological function deteriorates, the elderly's dependence on the auxiliary facilities of the sanitary system will increase. How the sanitary system can truly adapt to the needs and behavior habits of the elderly has also become a research focus. This article is based on demand theory, conducts user surveys and analysis of elderly bathroom space usage behaviors, and proposes the design of elderly home bathroom systems based on the relationship between people, products, and the environment. Through the adjustment of shower space, toilet space and washing space, improving the bathing experience of the elderly, and provides certain theoretical support and practical reference value for the follow-up research on the bathroom of the elderly.

\section{Acknowledgments}

This case comes from the "2019 Jiangsu Summer School of Arts Postgraduates" project with the theme of "Healthy Pension System Design" organized by Jiangnan University, Thank you very much to the following 6 classmates: Sun Aoran, Gu keyi, Su Min from Jiangnan University; Lin Chengpei from Fuzhou University and SARAH BOOT, SUMMER ASHBURY from Plymouth University in the UK. Without their joint efforts, the final project cannot be completed, sincere thanks!

\section{References}

1. Yang Shuyuan. (2015) Research on Sanitary Bathroom Design for the Elderly Based on Demand Theory. D. Master's Thesis of Chongqing University.

2. Zhang Xiaoxu. (2014) Consumer Demands and Marketing Strategies of Livable Communities for the Aged. D.Master's Thesis of Fujian Normal University. 
3. Deng Yuxia. (2019) Practice Difficulties and Solutions of "Internet +" Home-based Elderly Care Services. D. Master's Thesis of Changchun University of Technology.

4. Ge Jiahao. (2016) Architectural Design for the Elderly Based on Humanization. J. Anhui Architecture.

5. Si Wenqing. (2017) Study on the impact mechanism of basic medical insurance on the health of the elderly. D. Master Thesis of South China University of Technology.

6. Wei Juanjuan. (2013) Research on Design of Sanitary Home Bathroom System Based on Perceptual Image .D.Master's Thesis of Southwest Jiaotong University.

7. Li Zhen; Chen Feihu. (2008) Research on Barrierfree Design in the Living Environment of the Elderly J."Sichuan Architecture" .

8. Zhang Rupan. (2008) Research on Design of Urban Sanitary Home Bathroom System .D.Master's Thesis of Jiangnan University.

9. Tian Yan; Li Hong. (2009) Design of small swimming pool. J.Liaoning Building Materials.

10. Lin Zhilan. (2019) Suitable products" gradually entered the homes of ordinary people .J.Fujian Daily". 